\title{
Existencia de una medida invariante en algunas ecuaciones de evolución estocásticas
}

\author{
Claudio Fernando, Balcázar Huapaya ${ }^{1}$ \\ cbalcazarh@unmsm.edu.pe
}

\begin{abstract}
Resumen
Se estudia el proceso estocástico formado en base a la solución de una ecuación de evolución estocástica. Se presenta el teorema de la existencia de una medida invariante en el espacio de Banach de valores de las variables aleatorias, según se cumplan un conjunto de hipótesis en el proceso estocástico mencionado. El problema de valor inicial y frontera de la ecuación de la placa de Von Karman con término aleatorio de "Movimiento Browniano" es un ejemplo donde se construye una medida invariante.

Palabras Clave: Movimiento Browniano, medida invariante, ecuación de Von Karman estocástica.
\end{abstract}

\begin{abstract}
We study the stochastic process shaped with the solution of a stochastic evolution equation. We prove the theorem of the existence of a invariant measure in the Banach space of the values of the random variables, satisfying suitables hypothesis in the mentioned stochastic process. The initial-boundary value problem for the von Karman plate with random term of Brownian motion is a model where to build a invariant measure.
\end{abstract}

Keywords: Brownian motion, measure invariant, equation the Von Karman stochastic.

\section{Introducción}

Estudiaremos la existencia de una medida invariante para una cierta clase de ecuaciones de evolución estocásticas, y especificamente para la ecuación de Von Karman de la placa.

Las ecuaciones diferenciales determinísticas han sido estudiadas durante los últimos siglos, sin embargo los efectos aleatorios están presentes en cada caso real, esto conduce a las

\footnotetext{
${ }^{1}$ UNMSM, Facultad de Ciencias Matemáticas, Lima - Perú.
} 
ecuaciones diferenciales estocásticas, es decir ecuaciones diferenciales con procesos estocasticos y que nos proporcionan métodos para desarrollar modelos más cercanos a la realidad.

Si se prueba la existencia de una medida invariante en un cierto problema y la condición inicial tiene distribución de probabilidad igual a una medida invariante, entonces la distribución de probabilidad de la solución es invariante en el tiempo.

\section{Definiciones Preliminares}

Definición 2.1 Sea $(\Omega, \mathcal{U}, P)$ un espacio de probabilidad, es decir $\Omega$ es un conjunto de eventos elementales, $\mathcal{U}$ es una $\sigma$-algebra de subconjuntos de $\Omega$, y $P: \mathcal{U} \longrightarrow[0,1]$ es una medida de probabilidad.

Un proceso estocástico es una colección parametrizada de variables aleatorias $\{X(t) / t \in I\}$ definidas en el mismo espacio de probabilidad $(\Omega, \mathcal{U}, P)$, tomando valores en $\mathbb{R}^{n}$.

Definición 2.2 Una filtración es una familia creciente de $\sigma$-algebras de subconjuntos de $\Omega,\left\{\mathcal{F}_{s}: s \in I\right\}$ con $\mathcal{F}_{s} \subset \mathcal{F}_{t}, s \leq t$.

Definición 2.3 Un proceso $\{X(t): t \geq 0\}$, se dice adaptado a la filtración $\mathcal{F}_{t}$ si $X(t)$ es $\mathcal{F}_{t}-$ medible para todo $t \geq 0$. $\left(X(t)\right.$ es $\mathcal{F}_{t}$ - adaptado $)$.

Si $X(\cdot)$ es un proceso estocástico, para cada $t$ fijo, tenemos la variable aleatoria

$$
\omega \longmapsto X(t, \omega), \quad \omega \in \Omega
$$

Por otro lado, para cada $\omega \in \Omega$ fijo tenemos una trayectoria.

$$
t \longmapsto x(t, \omega), \quad t \in I
$$

Definición 2.4 Dado un espacio de probabilidad $(\Omega, \mathcal{U}, P)$ diremos que el proceso $(B(t))_{t>0}$ a valores en $\mathbb{R}^{n}$ es un movimiento Browniano estándar respecto de la filtración $\left(\mathcal{F}_{t}\right)_{t \geq 0}$ sobre el espacio $\Omega$ si es $\mathcal{F}_{t}$ - adaptado y satisface:

i) casi todas las trayectorias son continuas, es decir existe $\Omega_{0} \subset \Omega$ con $P\left(\Omega_{0}\right)=1$ tal que para todo $\omega \in \Omega_{0}$

$$
t \longmapsto B(t, \omega)
$$

es continua.

ii) $(B(t))_{t>0}$ tiene incremento con distribución normal: $\forall n \in \mathbb{N}$ y $t_{0}<t_{1} \ldots<t_{n}$ las variables aleatorias $B\left(t_{1}\right)-B\left(t_{0}\right), \ldots B\left({ }_{t n}\right)-B\left({ }_{t n-1}\right)$ son independientes con

$$
B\left(t_{i+1}\right)-B\left(t_{i}\right) \backsim N\left(0,\left(t_{i+1}-t_{i}\right) i d\right)
$$

iii) $B(0)=0$. 
Observamos que como $B(t)-B(s)$ es independiente de la $\sigma$-algebra $\mathcal{F}_{s}$, entonces

$$
E\left[B(t) \mathcal{F}_{s}\right]=B(s)
$$

en efecto,

$$
\begin{aligned}
E\left[B(t) \mathcal{F}_{s}\right] & =E\left[(B(t)-B(s))+B(s) \mid \mathcal{F}_{s}\right] \\
& =E\left[B(t)-B(s) \mid \mathcal{F}_{s}\right]+E\left[B(s) \mid \mathcal{F}_{s}\right] \\
& =E[B(t)-B(s)]+B(s) \\
& =B(s)
\end{aligned}
$$

Dado un proceso $X$, consideramos la evolución del mismo hasta un cierto tiempo aleatorio (tiempo de parada). Es importante observar que la decisión de parar, debe depender sólo de la información disponible hasta ese momento y no del futuro. O equivalemente si $\tau: \Omega \rightarrow \mathbb{R}^{+}$ es el tiempo de parada, el proceso $y(t):=X(t \wedge \tau)$ debe ser un nuevo proceso $\mathcal{F}_{t}$-adapatado.

Definición 2.5 Una variable aleatoria $\tau: \Omega \rightarrow \mathbb{R}$ se dice tiempo de parada si $\{\tau \leq t\}$; es $\operatorname{decir}\{\omega \in \Omega / \tau(\omega) \leq t\}$ es $\mathcal{F}_{t}$-medible para todo $t \geq 0$.

A continuación el siguiente teorema menciona algunas propiedades del movimiento Browniano. Cuya demostración se puede encontrar en [4])

Teorema 2.1 Sea $B(\cdot)$ un movimiento Browniano unidimensional definido en $(\Omega, \mathcal{U}, \mathbb{P})$, entonces:

1. Regularidad. Para todo $0<\gamma<\frac{1}{2}$ existe un conjunto $\Omega_{0}, P\left(\Omega_{0}\right)=1$ tal que para todo $\omega \in \Omega_{0}$ y para todo $T>0$ la función $B(\cdot, \omega)$ es uniformemente Hölder $\gamma$ en $[0, T]$.

2. No diferenciabilidad Para todo $\frac{1}{2}<\gamma \leq 1$ y casi todo $\omega \in \Omega$ la función $B(\cdot, \omega)$ no es Hölder $\gamma$ en ningún punto. En particular para casi todo $\omega \in \Omega$ la función $B(\cdot, \omega)$. no es derivable en ningún punto y no es de variación acotada en ningún intervalo.

3. Variación Cuadrática. Sea $0 \leq a<b$ supongamos que

$$
P^{n}=\left\{a=t_{0}^{n}<t_{1}^{n}<\cdots<t_{m_{n}}^{n}=b\right\}
$$

son particiones de $[a, b]$ tales que $\left|P^{n}\right| \rightarrow 0$ cuando $n \rightarrow \infty$. Entonces

$$
\sum_{k=0}^{m_{n}-1}\left[B\left(t_{k+1}^{n}\right)-B\left(t_{k}^{n}\right)\right]^{2} \rightarrow b-a
$$

en $L^{2}(\Omega)$

4. Rescale. Los procesos $\frac{1}{a} B\left(a^{2} t\right)$ y $\frac{1}{t} B\left(\frac{1}{t}\right)$ son movimientos Brownianos. 


\section{Existencia de medida invariante}

Sea $\left\{\Omega, \mathcal{F}, \mathcal{F}_{t}, P\right\}$ una base estocástica, es decir $P$ es una medida de probabilidad sobre el $\sigma$-algebra $\mathcal{F},\left\{\mathcal{F}_{t}\right\}$ es una filtración continua sobre $\mathcal{F}, \Omega$ es un conjunto de eventos elementales. $E(\cdot)$ denota la esperanza con respecto a $P$; supongamos que $X(t, s ; z), 0 \leq t \leq$ $s<\infty$ es una ruta, solución única de una cierta ecuación de evolución estocástica tal que $X(s, s ; z)=z$. En [5] se plantean las siguientes hipótesis a partir de las cuales se probará la existencia de una medida invariante para algunas ecuaciones de evolución estocásticas.

(I) Sea $\Xi$ un espacio de Banach separable.

$$
\begin{aligned}
X(\cdot, s ; z): \mathbb{R}^{+} & \longrightarrow \Xi \\
t & \longmapsto X(t, s, z)
\end{aligned}
$$

es un proceso continuo y adaptado a $\left\{\mathcal{F}_{t}\right\}_{t \geq s}$ para todo $z \in \Xi$.

Definimos la función

$$
\mathcal{P}(s, z, t, \Gamma)=P(X(t, s, z) \in \Gamma), \forall \Gamma \in \mathcal{B}(\Xi) \quad 0 \leq s \leq t<\infty, z \in \Xi,
$$

donde $\mathcal{B}(\Xi)$ es $\sigma$-algebra de Borel de $\Xi$. Asumimos:

(II) $\mathcal{P}(\cdot, \cdot ; \cdot, \cdot)$ es una función de probabilidad de transición homogénea en el tiempo, es decir satisface:

i) $\mathcal{P}(s, z ; t, \cdot)$ es una medida de probabilidad sobre $\{\Xi, \mathcal{B}(\Xi)\}$ para todo $z \in \Xi \mathrm{y}$ $0 \leq s<t<\infty$.

ii) $\mathcal{P}(s, z ; t, \Gamma)$ es $\mathcal{B}(\Xi)$-medible para todo $0 \leq s<t<\infty$ y $\Gamma \in \mathcal{B}(\Xi)$

iii) para todo $0 \leq s<t<\xi<\infty$ y $\Gamma \in \mathcal{B}(\Xi)$

$$
\mathcal{P}(s, z ; \xi, \Gamma)=\int_{\Xi} \mathcal{P}(s, z ; t, d y) \mathcal{P}(t, y ; \xi, \Gamma),
$$

iv) $\mathcal{P}(s, \cdot ; t, \cdot)=\mathcal{P}(s+h, \cdot ; t+h, \cdot)$ para todo $0 \leq s<t<\infty$ y $h>0$

(III) Existe un $z \in \Xi$ tal que

$$
E\left(\|X(t, 0 ; z)\|_{\Xi}\right) \leq M \text { para todo } t \geq 0
$$

y para alguna constante positiva $M$.

$(I V)$ Existe un espacio de Banach $\Upsilon$ tal que $\Xi \subset \Upsilon$, la inmersión $\Xi \rightarrow \Upsilon$ es continua, y cada bola cerrada de radio finito en $\Xi$ es un subconjuto compacto de $\Upsilon$. Además para cada función continua acotada $\psi$ sobre $\Xi$, existe una sucesión de funciones continuas $\left\{\psi_{k}\right\}_{k=1}^{\infty}$ sobre $\Upsilon$ tal que $\psi_{k}$ es uniformemente acotada en $k$ y

$$
\operatorname{Lim}_{k \rightarrow \infty} \psi_{k}(y)=\psi(y) \quad \text { para cada } y \in \Xi
$$


$(V)$ Para cada $0 \leq t<\infty$ fijo, y cada bola cerrada fija $S$ de radio finito en $\Xi$, si $\left\{z_{n}\right\}_{n=1}^{\infty}$ es una sucesión en $S$ tal que

$$
z_{n} \rightarrow z \text { en } \Upsilon
$$

entonces

$$
E\left(\phi\left(X\left(t, 0 ; z_{n}\right)\right)\right) \rightarrow E(\phi(X(t, 0 ; z)))
$$

para cada función continua acotada $\phi$ sobre $\Upsilon$.

Teorema 3.1. Bajo las hipotesis $(I)-(V)$ existe una medida invariante para el proceso $X(\cdot)$, es decir, existe una medida de probabilidad $\mu$ sobre $\Xi$ tal que

$$
\int_{\Xi} E(\psi(X(t, 0 ; y))) \mu(d y)=\int_{\Xi} \psi(y) \mu(d y)
$$

para todo $t \geq 0$ y para cada función continua acotada $\psi$ sobre $\Xi$

Prueba.

Elegimos $z \in \Xi$ y definimos la medida $\mu_{T}$; para todo $T>0$ por

$$
\mu_{T}(\Gamma)=\frac{1}{T} \int_{0}^{T} P(X(t, 0 ; z) \in \Gamma) d t
$$

y para cada $\Gamma \in \mathcal{B}(\Xi)$.

De la hipótesis $(I)$ :

Para cada función continua y acotada $\phi$ sobre $\Xi, E(\phi(X(t, 0 ; z)))$ es continua en $t$. Sea $\Gamma \subset \Xi, \Gamma$ cerrado. Entonces existe una sucesión de funciones continuas acotadas no negativas $\left\{\phi_{k}\right\}_{k=1}^{\infty}$ sobre $\Xi$ decrecientes tal que $\phi_{k}(y) \rightarrow \mathcal{X}_{\Gamma}(y)$ cuando $k \rightarrow \infty, \forall y \in \Xi$.

De donde

$$
E\left(\phi_{k}(X(t, 0 ; z))\right) \rightarrow E\left(\mathcal{X}_{\Gamma}(X(t, 0 ; z))\right)
$$

con $k \rightarrow \infty \forall t \geq 0$.

Aquí $P(X(\cdot, 0 ; z) \in \Gamma)$ es $\mathcal{B}([0, \infty])-$ medible.

Sea

$$
S=\{\Gamma \subset \Xi / P(X(\cdot, 0 ; z) \in \Gamma) \text { es un } \mathcal{B}([0, \infty])-\text { medible }\}
$$

$S$ incluye todos los subconjuntos cerrados de $\Xi$ y además $\mathcal{B}(\Xi) \subset S$.

Procedemos a definir

$$
\tilde{\mu}_{T}(\Gamma)=\mu_{T}(\Gamma \cap \Xi)
$$

para cada $\Gamma \in \mathcal{B}(\Upsilon)$.

Como la inmersión $\Xi \rightarrow \Upsilon$ es continua $\Gamma \cap \Xi$ es un subconjunto de Borel de $\Xi, \forall \Gamma \in \mathcal{B}(\Upsilon)$. $\tilde{\mu}_{T}$ es bien definida y es una medida de probabilidad sobre $\{\Upsilon, \mathcal{B}(\Upsilon)\}$.

Por la hipótesis $(I I I): \forall \varepsilon>0, \exists r_{\varepsilon}>0$ tal que

$$
P\left(\|X(t, 0 ; z)\|_{\Xi} \leq r_{\varepsilon}\right)>1-\varepsilon, \forall t \geq 0
$$


De la hipótesis $(I V)$ : Como $S_{y_{\varepsilon}}=\left\{y \in \Xi /\|y\|_{\Xi} \leq r_{\varepsilon}\right\}$ es compacto de $\Upsilon$, existe una sucesión $\left\{\tilde{\mu}_{T_{k}}\right\}_{k=1}^{\infty}$ y una medida de probabilidad $\tilde{\mu}$ sobre $\{\Upsilon, \mathcal{B}(\Upsilon)\}$ tal que $\underset{k \rightarrow \infty}{\operatorname{Lim}} T_{k}=\infty$ y

$$
\int_{\Upsilon} \phi(y) \tilde{\mu}_{T_{k}}(d y) \rightarrow \int_{\Upsilon} \phi(y) \tilde{\mu}(d y) \quad \text { cuando } k \rightarrow \infty
$$

para cada función continua y acotada $\phi$ sobre $\Upsilon$.

Como $S_{r_{\varepsilon}}$ es un subconjunto cerrado de $\Upsilon$, entonces

$$
1-\varepsilon \leq \operatorname{Lim}_{k \rightarrow \infty} \sup \tilde{\mu}_{T_{k}}\left(S_{r_{\varepsilon}}\right) \leq \tilde{\mu}\left(S_{r_{\varepsilon}}\right)
$$

Como $\varepsilon>0$ es arbitrario y cada subconjunto de Borel de $\Xi$ es también subconjunto de Borel de $\Upsilon, \tilde{\mu}(\Xi)=1$, y la restricción de $\tilde{\mu}$ a $\mathcal{B}(\Xi)$, denotado como $\mu$ es una medida de probabilidad sobre $\{\Xi, \mathcal{B}(\Xi)\}$. Esta es la medida sobre $\Xi$ que verifica la ecuación (3.1) del enunciado del teorema.

Elegimos una función $\phi: \Upsilon \rightarrow \mathbb{R}$ continua y acotada. Dado $\varepsilon>0 \exists r>0$ tal que

$$
\tilde{\mu}_{T_{k}}\left(S_{r}\right)=\mu_{T_{k}}\left(S_{r}\right)>1-\varepsilon, \forall k \geq 1 .
$$

Fijamos $t>0$ y sea

$$
f(y)=E(\phi(X(t, 0 ; y)))=\int_{\Xi} \mathcal{P}(0, y: t, d w) \phi(w) .
$$

Por la hipótesis $(V), f(y)$ es continua sobre $S_{r}$, con respecto a la norma de $\Upsilon$. Como $S_{r}$ es un subconjunto cerrado de $\Upsilon$, podemos extender $f$ a $\tilde{f}$ de $\Upsilon$ con la misma cota tal que $f(y)=\tilde{f}(y) ; \forall y \in S_{r}$.

Tenemos que:

$$
\begin{aligned}
\int_{\Upsilon} \tilde{f}(y) \tilde{\mu}_{T_{k}}(d y)= & \int_{\Upsilon-S_{r}} \tilde{f}(y) \tilde{\mu}_{T_{k}}(d y) \\
& +\int_{S_{r}} \tilde{\mu}_{T_{k}}(d y) \int_{\Xi} \mathcal{P}(0, y: t, d w) \phi(w)
\end{aligned}
$$

luego

$$
\begin{aligned}
& \mid \int_{S_{r}} \tilde{\mu}_{T_{k}}(d y) \int_{\Xi} \mathcal{P}(0, y: t, d w) \phi(w) \\
& -\int_{\Xi} \tilde{\mu}_{T_{k}}(d y) \int_{\Xi} \mathcal{P}(0, y: t, d w) \phi(w)<M \varepsilon \mid
\end{aligned}
$$

donde $M>0$ tal que $|\phi(y)| \leq M, \forall y \in \Upsilon$. 
De la hipótesis $(I I)$

$$
\begin{aligned}
& \int_{\Xi} \tilde{\mu}_{T_{k}}(d y) \int_{\Xi} \mathcal{P}(0, y: t, d w) \phi(w) \\
= & \frac{1}{T_{k}} \int_{0}^{T_{k}}\left(\int_{\Xi} \mathcal{P}(0, z: s, d y) \int_{\Xi} \mathcal{P}(0, y: t, d w) \phi(w)\right) d s \\
= & \frac{1}{T_{k}} \int_{0}^{T_{k}}\left(\int_{\Xi} \mathcal{P}(0, z: s+t, d y) \phi(y)\right) d s \\
= & \frac{1}{T_{k}} \int_{0}^{T_{k}+t}\left(\int_{\Xi} \mathcal{P}(0, z: \eta, d y) \phi(y)\right) d \eta
\end{aligned}
$$

Tenemos

$$
\begin{gathered}
\operatorname{Lim}_{k \rightarrow \infty}\left|\frac{1}{T_{k}} \int_{0}^{T_{k}+t}\left(\int_{\Xi} \mathcal{P}(0, z: \eta, d y) \phi(y)\right) d \eta-\int_{\Xi} \mu_{T_{k}}(d y) \phi(y)\right|=0, \\
\int_{\Xi} \mu_{T_{k}}(d y) \phi(y)=\int_{\Upsilon} \tilde{\mu}_{T_{k}}(d y) \phi(y)
\end{gathered}
$$

y

$$
\operatorname{Lim}_{k \rightarrow \infty} \int_{\Upsilon} \tilde{\mu}_{T_{k}}(d y) \phi(y)=\int_{\Upsilon} \tilde{\mu}(d y) \phi(y)=\int_{\Xi} \mu(d y) \phi(y)
$$

Luego

$$
\begin{gathered}
\operatorname{Lim}_{k \rightarrow \infty} \int_{\Upsilon} \tilde{f}(y) \tilde{\mu}_{T_{k}}(d y)=\int_{\Upsilon} \tilde{f}(y) \tilde{\mu}(d y) \\
\left|\int_{\Upsilon} \tilde{f}(y) \tilde{\mu}(d y)-\int_{S_{r}} f(y) \mu(d y)\right|<M \varepsilon \\
\int_{S_{r}} f(y) \mu(d y)=\int_{S_{r}} \mu(d y) E(\phi(X(t, 0 ; y))) \\
\left|\int_{S_{r}} \mu(d y) E(\phi(X(t, 0 ; y)))-\int_{\Xi} \mu(d y) E(\phi(X(t, 0 ; y)))\right|<M \varepsilon
\end{gathered}
$$

Se sigue de $(3,13)$ y $(3,6)$ que:

$$
\left|\int_{\Xi} \mu(d y) E(\phi(X(t, 0 ; y)))-\int_{\Xi} \mu(d y) \phi(y)\right|<4 M \varepsilon .
$$

Como $\varepsilon>0$ es arbitrario, tenemos

$$
\int_{\Xi} \mu(d y) E(\phi(X(t, 0 ; y)))=\int_{\Xi} \mu(d y) \phi(y)
$$

para toda función continua acotada $\phi$ sobre $\Upsilon$, para cada $t>0$. Elegimos cualquier función continua acotada $\psi$ sobre $\Xi$ y sea $\left\{\psi_{k}\right\}_{k=1}^{\infty}$ una sucesión en la hipótesis $(I V)$. 
Entonces, para cada $k \geq 1$, tenemos

$$
\int_{\Xi} \mu(d y) E\left(\psi_{k}(X(t, 0 ; y))\right)=\int_{\Xi} \mu(d y) \psi_{k}(y)
$$

haciendo tender $k \rightarrow \infty$, tenemos

$$
\int_{\Xi} \mu(d y) E(\psi(X(t, 0 ; y)))=\int_{\Xi} \mu(d y) \psi(y)
$$

esto completa la prueba.

El resultado de este teorema, se aplica a cierta clase de ecuaciones de evolución estocásticas, en particular a la ecuación de la placa de Von Karman estocástica, también se aplica a la ecuación de la onda en dimensión uno con una condición de frontera en términos del movimiento Browniano. (Ver [6]).

En lo que sigue se estudia la ecuación de la placa de Von Karman, como un típico ejemplo donde se contruye una medida invariante.

\section{La Ecuación de Von Karman Estocástica}

Consideremos el problema de valor inicial y frontera para la ecuación de la placa de Von Karman con término estocástico.

$$
\begin{gathered}
u_{t t}+\Delta^{2} u-[u, v]=g \frac{d B}{d t} \text { en }(0, T) \times G \\
\Delta^{2} v+[u, u]=0 \text { en }(0, T) \times G \\
u=\frac{\partial u}{\partial \eta}=0, v=\frac{\partial v}{\partial \eta}=0 \text { sobre }[0, T] \times \partial G \\
u=u_{0}(x), u_{t}=u_{1}(x), \text { en } t=0
\end{gathered}
$$

donde $g \in L^{2}(G), G$ es un dominio acotado en $\mathbb{R}^{2}$ con frontera regular $\partial G, \Delta^{2}$ es el operador biarmónico, $B$ es un movimiento Browniano que es un proceso estocástico, y el corchete es definido por

$$
[u, v]=u_{x x} v_{y y}+v_{x x} u_{y y}-2 u_{x y} v_{x y}
$$

Observación 4.1. Consideremos una base ortonormal completa $\left\{\phi_{k}\right\}_{k=1}^{\infty}$ para $L^{2}(G)$ donde cada $\phi_{k}$ es una autofunción de

$$
\begin{aligned}
\Delta^{2} \phi_{k} & =\lambda_{k} \phi_{k} \text { en } G \\
\phi_{k} & =\frac{\partial \phi_{k}}{\partial \eta}=0 \quad \text { sobre } \partial G
\end{aligned}
$$


Definimos el operador $\mathcal{G}$ sobre $H^{-2}(G)$ por

$$
\mathcal{G} h=\sum_{k=1}^{\infty} \frac{1}{\lambda_{k}} a_{k} \phi_{k} \text { para } h=\sum_{k=1}^{\infty} a_{k} \phi_{k} \in H^{-2}(G) .
$$

Se tiene que $\mathcal{G}$ es la inversa de $\Delta^{2}$ con las condiciones de frontera

$$
\phi=\frac{\partial \phi}{\partial \eta}=0 \text { sobre } \partial G
$$

Se tiene que para todo $f, g \in L^{2}(G)$ :

$$
\begin{aligned}
|\langle f, \mathcal{G} g\rangle| & \leq\|f\|_{H^{-2}(G)}\|g\|_{H^{-2}(G)} \\
\langle f, \mathcal{G} g\rangle & =\|f\|_{H^{-2}(G)}^{2},
\end{aligned}
$$

$\mathrm{y}$

$$
\|\mathcal{G}[f, g]\|_{W^{2, \infty}(G)} \leq c\|f\|_{H^{-2}(G)}\|g\|_{H^{-2}(G)}
$$

\subsection{Teorema de Existencia de la Ecuación de Von Karman es- tocástica}

Tenemos que $B$ es un movimento Browniano sobre la base estocástica $\left\{\Omega, \mathcal{F}, \mathcal{F}_{t}, P\right\}$ donde $P$ es una medida sobre el $\sigma$-algebra $\mathcal{F},\left\{\mathcal{F}_{t}\right\}$ es una filtración continua por la derecha sobre $\mathcal{F}$. $E(\cdot)$ denota la esperanza con respecto a $P$. Cuando $V$ es un espacio de Banach $\mathcal{B}(V)$ denota el conjunto de todos los subconjuntos de Borel de $V$ y una función $h: \Omega \rightarrow V$ se dice $\mathcal{F}$ - medible si $h^{-1}(\mathcal{O}) \in \mathcal{F}, \forall \mathcal{O} \in \mathcal{B}(V)$. Para $1 \leq p<\infty, L^{P}(\Omega ; V)$ denota el conjunto de todas la funciones $h: \Omega \rightarrow V$ medible fuertemente respecto a $\mathcal{F}$ tal que

$$
\int_{\Omega}\|h\|_{V}^{P} d P<\infty
$$

Teorema 4.1 Para todo $t>0 y\left(u_{0}, u_{1}\right) \in H_{0}^{2}(G) \times L^{2}(\Omega)$, existe una única solución $u$ de $(4,1)-(4,4)$ tal que $\left(u, u_{t}\right)$ es adaptado a $\left\{\mathcal{F}_{t}\right\} y$

$$
\left(u, u_{t}\right) \in L^{2}\left(\Omega ; C[0, T] ; H_{0}^{2}(G) \times L^{2}(G)\right)
$$

aquí u satisface ( 4.1 ) en el sentido siguiente:

Para casi todo $w \in \Omega$ vale que:

$$
\begin{aligned}
& \left\langle u_{t}\left(t_{2}\right), \psi\right\rangle-\left\langle u_{t}\left(t_{1}\right), \psi\right\rangle+\int_{t_{1}}^{t_{2}}\langle\Delta u, \Delta \psi\rangle d t \\
& +\int_{t_{1}}^{t_{2}}\langle[u, \mathcal{G}[u, u]], \psi\rangle d t=\int_{t_{1}}^{t_{2}}\langle g, \psi\rangle d B
\end{aligned}
$$

para todo $\psi \in H_{0}^{2}(G), o \leq t_{1}<t_{2} \leq T$ y $\mathcal{G}$ es el inverso del operador $\Delta^{2}$. 
Prueba. Ver [5].

Para la prueba de este teorema se usan resultados previos de [1], [2], [3]

Observación 4.2. Cada $\left(u, u_{t}\right)$ es adaptado a $\left\{\mathcal{F}_{t}\right\}$. Podemos deducir que:

$$
E\left(\sup _{0 \leq t \leq T}\left(\left\|u_{t}(t)\right\|_{L^{2}(G)}^{2}+\|\Delta u(t)\|_{L^{2}(G)}^{2}+\|\Delta v(t)\|_{L^{2}(G)}^{2}\right)\right) \leq k_{0}
$$

para alguna constante $k_{0}$, para todo $T>0$ donde $v=\mathcal{G}[u, u]$. (Ver [5])

Para la prueba de unicidad de la solución suponemos que $\left(\tilde{u}, \tilde{u}_{t}\right)$ es otra solución de $(4,1)-(4,4)$ en $L^{2}\left(\Omega ; C\left([0, T] ; H_{0}^{2}(G) \times L^{2}(G)\right)\right)$.

Entonces $u-\tilde{u}$ satisface

$$
u_{t t}-\tilde{u}_{t t}+\Delta^{2}(u-\tilde{u})+[u, \mathcal{G}[u, u]]-[\tilde{u}, \mathcal{G}[\tilde{u}, \tilde{u}]]=0
$$

para casi todo $w \in \Omega$. Como $\left(u, u_{t}\right)$ y $\left(\tilde{u}, \tilde{u}_{t}\right)$ pertenecen a

$$
C\left([0, T] ; H_{0}^{2}(G) \times L^{2}(G)\right)
$$

para casi todo $w$, podemos aplicar el mismo argumento como el caso determinístico para concluir que $u=\tilde{u}$ para casi todo $w \in \Omega$. 4.1.

Fijamos $\lambda$ tal que $0<\lambda<\operatorname{mín}\left(1, \lambda_{1}\right)$ donde $\lambda_{1}$ es el primer autovalor en la observación

Definimos

$$
Q(t)=\left\|u_{t}(t)\right\|_{L^{2}(G)}^{2}+\|\Delta u(t)\|_{L^{2}(G)}^{2}+\|\Delta v(t)\|_{L^{2}(G)}^{2}+\lambda\left\langle u_{t}(t), u(t)\right\rangle
$$

entonces $E(Q(t)) \leq C_{M}$ para alguna constante $C_{M}$. Ver [5]. De aquí se deduce que

$$
E\left(\|X(t, 0 ; z)\|_{H^{2}(G) \times L^{2}(G)}\right) \leq M, \quad \forall t \geq 0
$$

y para alguna constante $M>0$.

Tomamos cualquier $s>0$ como el tiempo inicial y $\zeta=\left(\zeta_{0}, \zeta_{1}\right)$ como el valor inicial para el problema de Cauchy ( 4.1$)$ - ( 4.4$)$ con valores de $\zeta$ en $H_{0}^{2}(G) \times L^{2}(G)$ y $\mathcal{F}_{s}$-medible tal que

$$
\zeta \in L^{2}\left(\Omega ; H_{0}^{2}(G) \times L^{2}(G)\right) \quad \text { y } \mathcal{G}\left[\zeta_{0}, \zeta_{0}\right] \in L^{2}\left(\Omega ; H_{0}^{2}(G)\right) .
$$

Escribimos $X(t, s ; \zeta)=\left(u(t), u_{t}(t)\right)$ donde $u$ es la solución de (4.1) - (4.3) para $t \geq s$ satisfaciendo $\left(u(s), u_{t}(s)\right)=\zeta$.

Entonces para $T>s$

$$
X(\cdot, s ; \zeta) \in L^{2}\left(\Omega ; C\left([s, T] ; H_{0}^{2}(G) \times L^{2}(G)\right)\right)
$$

es decir

$$
\int_{\Omega}\|X(\cdot, s ; \zeta)\|_{C\left([s, T] ; H_{0}^{2}(G) \times L^{2}(G)\right)}^{2} d P<\infty
$$


entonces

$$
\int_{\Omega} \operatorname{máx}_{s \leq t \leq T}\|X(t, s ; \zeta)\|_{H_{0}^{2}(G) \times L^{2}(G)}^{2} d P<\infty
$$

Por otra parte para

$$
\begin{aligned}
0 & \leq s<t, z \in H_{0}^{2}(G) \times L^{2}(G) \\
\text { у } \Gamma & \in \mathcal{B}\left(H_{0}^{2}(G) \times L^{2}(G)\right),
\end{aligned}
$$

definimos

$$
\mathcal{P}(s, z ; t, \Gamma)=P(X(t, s ; z) \in \Gamma)
$$

Lema 4.1 Elegimos cualquier función continua acotada

$$
\psi: H_{0}^{2}(G) \times L^{2}(G) \rightarrow \mathbb{R}, \quad t>s \geq 0
$$

entonces

$$
E(\psi(X(t, s ; z)))=\int_{H_{0}^{2}(G) \times L^{2}(G)} \mathcal{P}(s, z ; t, d y) \psi(y)
$$

es continua en $z \in H_{0}^{2}(G) \times L^{2}(G)$.

Prueba. Ver [5]

Observación 4.3. Sea $\Xi=H_{0}^{2}(G) \times L^{2}(G)$, el lema anterior implica $\mathcal{P}(s, \cdot ; t, \Gamma)$ es $\mathcal{B}(\Xi)$ medible para $0 \leq s<t<\infty$ y $\Gamma \in \mathcal{B}(\Xi)$.

Lema $4.2 X(\cdot)$ tiene la propiedad de Markov, y la función de transición probabilística es homogénea en el tiempo.

Prueba. Ver [5]

Lema 4.3 Sea $S_{L}=\left\{y \in H_{0}^{2}(G) \times L^{2}(G) /\|y\|_{H_{0}^{2}(G) \times L^{2}(G)} \leq L\right\}$, y sea $\left\{z_{n}\right\}$ una sucesión en $S_{L}$ tal que $z_{n} \rightarrow z$ en $H_{0}^{1}(G) \times H^{-1}(G)$. Si $\phi$ es cualquier función continua acotada en $H_{0}^{1}(G) \times H^{-1}(G)$, entonces para todo $t>0$

$$
E\left(\phi\left(X\left(t, 0, z_{n}\right)\right)\right) \rightarrow E(\phi(X(t, 0 ; z)))
$$

Prueba. Ver [5].

Lema 4.4 Sea $\psi$ una función continua acotada sobre. Entonces existe una sucesión $\left\{\psi_{k}\right\}$ de funciones continuas sobre $H_{0}^{1}(G) \times H^{-1}(G)$ acotadas uniformemente en $k$ y además

$$
\psi_{k}(y) \rightarrow \psi(y) \quad \text { cuando } k \rightarrow \infty
$$

para cada $y \in H_{0}^{2}(G) \times L^{2}(G)$.

Prueba. Ver [5] 
Teorema 4.2 Existe una medida invariante sobre $H_{0}^{2}(G) \times L^{2}(G)$ para el problema ( 4.1 ) - ( 4.4$)$.

Prueba.

En efecto: Sea $\Xi=H_{0}^{2}(G) \times L^{2}(G)$ y $\Upsilon=H_{0}^{1}(G) \times H^{-1}(G)$. Se tiene que $\Upsilon$ es un espacio de Banach y que $\Xi \subset \Upsilon$ y además la inmersión $\Xi \rightarrow \Upsilon$ es continua. Las hipótesis $(I)-(V)$ de la sección 3 se cumple por los lemas anteriores con lo cual se prueba este teorema.

Observación 4.4. Algunos resultados generales sobre medidas invariantes en ecuaciones de evolución estocásticas son presentadas en [1] y [2] , las cuales pueden cubrir una amplia clase de ecuaciones de evolución incluyendo la ecuación de onda.

En [6] tenemos el problema de la ecuación de onda unidimensional con condición aleatoria en la frontera:

$$
\begin{aligned}
u_{t t} & =a u_{x x}-b u_{t}, a>0, b>0 \quad(x, t) \in(0, L) \times(0, T) \\
u_{x}(0, t) & =\frac{d B}{d t}, \quad u(L, t)=0 \quad t \in(0, T) \\
u(x, 0) & =u_{0}(x), \quad u_{t}(x, 0)=u_{1}(x), \quad x \in(0, L)
\end{aligned}
$$

donde $B$ es un "movimiento Browniano".

Se considera el operador $\Lambda=-\frac{d^{2}}{d x^{2}}$ en $L^{2}(0, L)$, con dominio

$$
D(\Lambda)=\left\{\varphi \in H^{2}(0, L) / \varphi_{x}(0)=0, \varphi(L)=0\right\}
$$

y sean $\left\{\lambda_{k}\right\}_{k=1}^{\infty},\left\{\varphi_{k}\right\}_{k=1}^{\infty}$ sucesiones de todos los autovalores y las correspondientes autofunciones normalizadas para $(\Lambda, D(\Lambda))$

ie.

$$
\left\{\begin{array}{l}
\Lambda \varphi_{k}=\lambda_{k} \varphi_{k}, \quad 0<x<L \\
\frac{d \varphi_{k}}{d x}(0)=0, \quad \varphi_{k}(L)=0
\end{array}\right.
$$

se define para $\theta \in \mathbb{R}$

$$
H_{\dagger}^{\theta}=\left\{f=\sum_{k=1}^{\infty} a_{k} \varphi_{k} / \sum_{k=1}^{\infty} \lambda_{k}^{\theta}\left|a_{k}\right|^{2}<\infty\right\}
$$

el cual es un espacio de Hilbert con un obvio producto interno. En [6] se prueba que existe una medida invariante para ( 4.8$),(4.10)$ sobre

$$
\left\{L^{2}(0, L) \times H_{\dagger}^{-1}, \mathcal{B}\left(L^{2}(0, L) \times H_{\dagger}^{-1}\right)\right\}
$$




\section{Conclusión}

Dado el problema de valor inicial y frontera para la ecuación de la placa de Von-Karman, como es formulado en (4.1) - (4.4) ; si $u$ es la solución respectiva, y definimos el proceso estocástico $X(t, s ; \zeta)=\left(u, u_{t}\right)$, se puede construir una medida invariante $u$ en el espacio $H_{0}^{2}(G) \times L^{2}(G)$ tal que se verifica la ecuación (3.1) del teorema 3.1. Con una medida invariante, si la distribución de la condición inicial tiene distribución de probabilidad igual a una medida invariante, entonces la distribución de probabilidad de la solución es invariante en el tiempo.

\section{Referencias}

[1] DA PRATO G. AND ZABCZYB J., Stochastic Equation in infinite Dimensions, Cambridge University Press, Cambridge. 1992

[2] DA PRATO G. AND ZABCZYB J., Ergodicity for infinite Dimensinal Systems, Cambridge University Press, Cambridge. 1996

[3] EVANS L., An Introduction to Stochastic Diferencial Equations. Departament of Mathematics UC Berkeley. (http://math.berkeley.edu/ evans/SDE.course.pdf)

[4] KARATZAS I. AND SHREVE S., Brownian Motion and Stochastic Calculus. 2nd ed. Springer, New York, Berlin, Heidelberg, 1997.

[5] KIM, J. U. Invariant measures for the Stochastic Von Karman plate equation. SIAM J. Math. Anal 36 . 1689-1703, 2005

[6] KIM, J. U. A One-Dimensional Wave Equation with white Noise Boundary Condition. Applied Mathematics Optimization. Springer Science + Business Media. inc, 2006 